\title{
Features of Otolithic Dysfunction in Patients with Sudden Sensorineural Hearing Loss at Different Ages
}

\author{
Xiaorong Niu', Qing Zhang1*, Xinda Xu',2, Peng Han1, Ying Cheng1, Ying Gao1, Rui Zhang1,3, \\ Yintong Yang1,4, Zichen Chen'1, Juan Hu1, Yanfei Chen', Min Xu1 ${ }^{*}$ \\ ${ }^{1}$ Department of Otorhinolaryngology Head and Neck Surgery, Ear Institute, Second Affiliated Hospital, Xi'an \\ Jiaotong University College of Medicine, Xi'an, China \\ ${ }^{2}$ EYE and ENT Hospital of Fudan University, Shanghai, China \\ ${ }^{3}$ Department of Otolaryngology, Xi'an Children's Hospital, Xi'an, China \\ ${ }^{4}$ Department of Otolaryngology, Xi'an Fourth People's Hospital, Xi'an, China \\ Email: ${ }^{*}$ ent551205@163.com, zhqent@163.com
}

Received 7 September 2015; accepted 22 September 2015; published 25 September 2015

Copyright (C) 2015 by authors and Scientific Research Publishing Inc.

This work is licensed under the Creative Commons Attribution International License (CC BY).

http://creativecommons.org/licenses/by/4.0/

c) (7) Open Access

\begin{abstract}
Objective: To observe the otolithic function in sudden sensorineural hearing loss (SSHL) patients in different age groups by using vestibular evoked myogenic potentials (VEMPs). Methods: One hundred and seventy unilateral SSHL patients were divided into 5 age groups. The 170 affected ears belonged to study group. The opposite healthy ears of 170 patients and 138 normal ears of 69 age- and sex-matched normal people at different ages were set as control groups. Ocular VEMP (oVEMP) and cervical VEMP (cVEMP) both evoked by air-conducted sound (ACS) were employed for vestibular otolithic function assessment. Results: The response rates of cVEMP and oVEMP were lower and abnormal rates were higher in affected ears of SSHL patients compared to the opposite healthy ears and normal control ears in all age groups, except patients over 60 years. The response rates of VEMPs significantly declined with increasing age, not only in normal control ears, but also in affected and healthy ears of SSHL patients. But, the abnormal rates of VEMPs showed no significant difference in affected ears at different ages. Conclusions: Dysfunction of vestibular otolithic organs in SSHL patients presented respective features at different ages. It is necessary to consider the age when the clinicians determine the otolithic condition of patients based on the VEMPs.
\end{abstract}

\section{Keywords}

Ocular Vestibular Evoked Myogenic Potential (oVEMP), Cervical Vestibular Evoked Myogenic Potential (cVEMP), SSHL, Age, Otolithic Function

\footnotetext{
${ }^{*}$ Corresponding author.
}

How to cite this paper: Niu, X.R., Zhang, Q., Xu, X.D., Han, P., Cheng, Y., Gao, Y., Zhang, R., Yang, Y.T., Chen, Z.C., Hu, J., Chen, Y.F. and Xu, M. (2015) Features of Otolithic Dysfunction in Patients with Sudden Sensorineural Hearing Loss at Different Ages. Journal of Biosciences and Medicines, 3, 1-8. http://dx.doi.org/10.4236/jbm.2015.310001 


\section{Introduction}

Sudden sensorineural hearing loss (SSHL) is defined as a sudden onset of more than $30 \mathrm{~dB}$ sensorineural hearing loss in at least three consecutive audiometric frequencies for unknown causes, which occurs within 3 days or less [1]. Several national surveys have estimated the incidence of SSHL at between five and 30 cases per 100,000 per year [2]-[4]. However, the German guideline of SSHL in 2011 has shown an incidence as high as 160 - 400 cases per 100,000 per year [5]. The etiology and pathophysiological mechanisms of SSHL have not been identified, although viral infection, vascular obstruction, and autoimmune reaction have been proposed [6]. And tension, pressure, mood swings, irregular life, and sleep disorders are generally considered as the main inducing factors of SSHL [7]. Vestibular symptoms are reported to be present in $28 \%$ to $57 \%$ of patients [8]. With the emphasis on vestibular function assessment, many researchers have studied the features of vestibular disorders in SSHL patients. In recent studies, it was found that vestibular otolith organs could be impaired in SSHL patients, and the otolithic dysfunction could be detected by vestibular evoked myogenic potentials (VEMPs) [9][11].

VEMPs are recorded from the superficial skeletal muscles in response to powerful suitable stimuli, such as air-conducted sound (ACS), bone-conducted vibration (BCV) or electrical stimulation. It has been used as clinical tests to assess vestibular macula function including saccule and utricle and the integrity of their afferent pathways for around 20 years. Cervical VEMP (cVEMP), which is recorded from the surface of sternocleidomastoid muscle (SCM) and generated by the activation of saccular afferents, reflects the function of the saccule and inferior vestibular nerve input pathway [12]. Ocular VEMP (oVEMP) is considered able to reflect the function of utricle and the superior vestibular nerve input pathway, which is recorded from the skin surface of contralateral inferior oblique muscle (beneath the eyes) [13]. In our previous study, cVEMP and oVEMP responses of 194 healthy ears have been collected, and we found that age might be an important influence on the presence and waveform features of VEMPs [14].

The clinical features such as manifestation and recovery of SSHL patients at different ages have already been reported in many researches, with, to our knowledge, none investigating differences of vestibular otolithic dysfunction in patients between different ages. Thus, in the present study, we observed the function of otolithic organs and their input pathways in SSHL patients at different ages using ACS-cVEMP and ACS-oVEMP test, hoping to provide some reference for the clinicians when they evaluate the otolithic condition of patients based on the VEMPs responses.

\section{Materials and Methods}

\subsection{Clinical Data}

This study retrospectively evaluated patients with unilateral SSHL, who visited the Department of Otorhinolaryngology of Second Affiliated Hospital of Xi'an Jiaotong University School of Medicine from May 2011 to May 2015. The inclusion criteria of SSHL patients (referred to the SSHL guideline established by AAO-HNS in 2012 [1]): (1) unilateral unfluctuating sensorineural hearing loss with sudden onset (within 3 days or less); (2) hearing loss of more than $30 \mathrm{~dB}$ at three consecutive audiometric frequencies; (3) no identifiable cause; (4) absence of other neurological signs. Exclusion criteria: (1) history of traumatic brain injury, otitis media, vertigo, tinnitus or hearing loss, etc; (2) central or retrocochlear hearing loss as measured by auditory brainstem response and MRI, such as acoustic neuroma; (3) conductive hearing impairment, which may affect the results of the vestibular tests; (4) over 30 days from the onset of hearing loss.

One hundred and seventy patients (mean age $43.75 \pm 13.80$ years; range 10 - 74 years, female/male 90/80) were involved as Study Group in our study. They were divided into 5 groups according to age, age $\leq 30$ years as group 1, 31 - 40 years as group 2, 41 - 50 years as group 3, 51 - 60 years as group 4 and $\geq 61$ years as group 5 . The average PTA of 170 affected ears was $82.74 \pm 21.30 \mathrm{~dB}$ HL, ranging from $41 \mathrm{~dB}$ to $126 \mathrm{~dB}$ HL. Ninety-six $(n=170,56.5 \%)$ of them suffered from vertigo or dizziness. All patients underwent audiometry, ACS-cVEMP and ACS-oVEMP tests.

The opposite healthy ears of 170 SSHL patients and 69 age- and sex-matched normal people (mean age 45.81 \pm 16.97 years; range 10 - 78 years, female/male 41/28) were recruited as Control Groups in this study. One hundred and seventy healthy ears and 138 normal ears were collected and tested oVEMP and cVEMP.

The study was approved by the regional ethical standards committee in the Second Affiliated Hospital of Xi'an Jiaotong University School of Medicine. Each subject signed the informed consent form for participation. 


\subsection{Audiometry}

The hearing level of each patient was obtained on the first visit. The pure-tone average (PTA) was calculated as the average threshold at 500,1 k, $2 \mathrm{k}$ and $4 \mathrm{k} \mathrm{Hz}$. If the hearing threshold at a certain frequency was off the scale, the estimated threshold of the frequency will be the maximum sound level generated by the audiometer plus 10 dB HL for calculating.

\subsection{VEMPs Testing}

The specific test methods were described in previous study [15] [16]. Briefly, each subject lay in the supine position. In cVEMP testing, two active electrodes were placed in the middle of the bilateral sternocleidomastoid (SCM) muscles; two reference electrodes were placed above the bilateral sternoclavicular joints and a ground electrode on the midline of the forehead. Interelectrode resistance should be less than $5 \mathrm{k} \Omega$. Each subject was required to raise her/his head off the pillow to activate the SCM and keep the head in the centerline of the body when sounds were presented through an insert earphone [15]. In oVEMP testing, two active electrodes were placed $1 \mathrm{~cm}$ below the lower lid margin of each eye in line with the pupil, the reference electrodes were placed 1 $\mathrm{cm}$ below each active electrodes and a ground electrode on the midline of the forehead. Interelectrode resistance should be less than $5 \mathrm{k} \Omega$. Each subject was asked to look upwards when hearing sound from the insert earphone [16].

Air-conducted sound (ACS) with $500 \mathrm{~Hz}$ short tone burst (rise/fall time $=1 \mathrm{~ms}$; plateau time $=2 \mathrm{~ms}$ ) was used for examination. The electromyographic signal from the stimulated side was amplified using a GN Otometrics (Taastrup, Denmark) ICS ChartrEP analyser. The stimulation rate was 5/s, and the responses to 50 stimuli were averaged. ACS was transmitted through a calibrated insert earphone. A stimulus level of $131 \mathrm{~dB}$ SPL was used as the default starting intensity to check whether the subject's VEMPs could be elicited and to identify the waveforms. The stimulus intensity would decrease in step of $10 \mathrm{~dB}$ or increase $5 \mathrm{~dB}$, depending upon the presence or absence of VEMPs, respectively.

\subsection{Observation Index for cVEMP and oVEMP}

VEMPs were obtained when there were reproducible short-latency biphasic waveforms appearing. Unrecognizable or unrepeatable waveforms were regarded as absence of responses. The lowest stimulus intensity of an identifiable and repeatable biphasic wave was recorded as the threshold (dB SPL). Parameters such as P1 and N1 latencies (ms), interpeak latency (ms), and amplitude $(\mu \mathrm{V})$ were recorded at a stimulus level of $131 \mathrm{~dB}$ SPL. The latencies of P1 and N1 were determined by the time between 0 ms and the peaks of P1 and N1. Interpeak latency was an absolute value of the time between P1 and N1. Amplitude was defined as the vertical distance between the peaks of P1 and N1.

Normal values of parameters at a stimulus level of $131 \mathrm{~dB}$ SPL of oVEMP and cVEMP in different age groups among Chinese population have been recorded in our previous study [14]. Normal ranges of those parameters in different age groups were calculated as Mean \pm 2 SD, respectively. The absence of VEMPs and recorded parameter values out of the normal ranges, such as elevated thresholds and prolonged latencies, were considered abnormalities in patients with SSHL.

\subsection{Statistical Analysis}

Data was analyzed using SPSS Statistics 13.0. The $\chi^{2}$ test or Fisher's Exact Test was used for comparison of the abnormal and response rates. Hearing thresholds were analyzed by Independent-Samples $T$ test. The level of statistical significance was set at $P<0.05$.

\section{Results}

\subsection{Clinic Features of SSHL Patients in Every Age Group}

As shown in Table 1, one hundred and seventy SSHL patients were divided into five groups by age. From group 1 to 5 , there were 30 patients, 33 patients, 53 patients, 35 patients and 19 patients belonged to each group respectively. The sex ratio, distribution of affected sides, course of disease, incidence of vertigo, and hearing level of the affected ear were similar among 5 groups $(P>0.05)$. Most patients visited our hospital in 1 or 2 weeks 
Table 1. Clinic features of SSHL patients in every age group.

\begin{tabular}{|c|c|c|c|c|c|c|c|c|c|c|c|}
\hline \multirow{2}{*}{ Age groups } & \multirow{2}{*}{$\mathrm{n}$} & \multicolumn{2}{|c|}{ Sex (n) } & \multicolumn{2}{|c|}{ Side (n) } & \multicolumn{3}{|c|}{ Disease course (n) } & \multirow{2}{*}{$\begin{array}{c}\text { PTA } \\
\text { (dB HL) }\end{array}$} & \multicolumn{2}{|c|}{ Vertigo (n) } \\
\hline & & M & $\mathrm{F}$ & $\mathrm{L}$ & $\mathrm{R}$ & $\leq 1 \mathrm{w}$ & $1 w-2 w$ & $>2 \mathrm{w}$ & & With & Without \\
\hline 1 & 30 & 14 & 16 & 18 & 12 & 19 & 6 & 5 & $78.33 \pm 20.05$ & 18 & 12 \\
\hline 2 & 33 & 18 & 15 & 13 & 20 & 21 & 8 & 4 & $82.45 \pm 15.70$ & 14 & 19 \\
\hline 3 & 53 & 28 & 25 & 23 & 30 & 37 & 12 & 4 & $80.90 \pm 22.35$ & 32 & 21 \\
\hline 4 & 35 & 15 & 20 & 15 & 20 & 25 & 4 & 6 & $89.18 \pm 22.80$ & 18 & 17 \\
\hline 5 & 19 & 5 & 14 & 7 & 12 & 10 & 5 & 4 & $83.49 \pm 24.98$ & 14 & 5 \\
\hline Total & 170 & 80 & 90 & 76 & 94 & 112 & 35 & 23 & $82.74 \pm 21.30$ & 96 & 74 \\
\hline
\end{tabular}

after onset, only $13.53 \%$ (23/170) of patients came to the hospital over 2 weeks. The average hearing loss was about $80 \mathrm{~dB}$ HL and patients suffered from vertigo were roughly the same amount to whom did not (Table 1).

\subsection{Response and Abnormal Rates of VEMPs between Affected, Healthy and Normal Control Ears in Every Age Group}

In total 170 SSHL patients, the oVEMP revealed presence in 85 affected ears (50\%) and abnormal responses in 98 affected ears (57.6\%). The cVEMP waves were elicited in 91 affected ears (53.5\%) and abnormal responses were observed in 92 affected ears (54.1\%). Except group 5, the response rates of cVEMP and oVEMP were lower and abnormal rates were higher in affected ears of SSHL patients compared to the opposite healthy ears and normal control ears in all groups (Table 2). And, although the response rate of cVEMP in group 1 didn't show significant differences in statistical analysis between affected, healthy and normal control ears, we still can see the numerical trend of lower response rate in affected ears (Table 2).

\subsection{The Effect of Age on Response and Abnormal Rates of VEMPs in Affected, Healthy and Normal Control Ears}

The response rates of cVEMP and oVEMP significantly declined with increasing age $\left(P<0.01, \chi^{2}\right.$ test) in all affected, healthy and normal control ears. The abnormal rates of VEMPs in normal control ears increased along with age $\left(P<0.01, \chi^{2}\right.$ test). Abnormal cVEMP and oVEMP only showed an increasing trend in both affected and healthy ears, except abnormal rate of oVEMP in healthy ears rises significantly with age $\left(P<0.05, \chi^{2}\right.$ test $)$ (Figure 1).

\section{Discussion}

As shown in Table 1, the peak incidence of SSHL was between 40 to 50 years old and the mean age of SSHL patients was $43.75 \pm 13.80$ years. Similar percentages of males and females were found among 170 SSHL patients, and left and right sides were affected almost equally $(P>0.05)$. In present study, there were $56.5 \%$ patients suffered from vertigo and the average PTA of 170 patients was $82.74 \pm 21.30 \mathrm{~dB}$ HL. These clinic features were similar with previous reports [8] [11]. Overall, there had no significant differences on above clinic features among 5 age groups.

It has been reported in many studies that there could be a particular percentage of otolithic involvement while cochlear impairment in patients with SSHL [9]-[11] [17]. As objective testing programmes, cVEMP and oVEMP tests were employed to assess saccular and utricular fuction by researchers. You et al. reported abnormal rates of $47 \%$ in cVEMP test and 48\% in oVEMP test in 75 unilateral SSHL patients (mean age $54 \pm 16$ years) [11]. Zhang et al. found that oVEMP and cVEMP responses were observed in $40.0 \%$ and $62.5 \%$ ears among 40 SSHL patients (mean age $48.4 \pm 14.1$ years, range 12 - 78 years) [9]. Our study showed that the oVEMP revealed abnormal responses in 57.6\% patients, and the abnormal responses of cVEMP were observed in $54.1 \%$ patients, similar to previous studies.

In our previous study, we collected cVEMP and oVEMP results in 194 healthy ears and found that the response rates decreased, thresholds elevated, amplitudes deceased and latencies prolonged in both examinations 

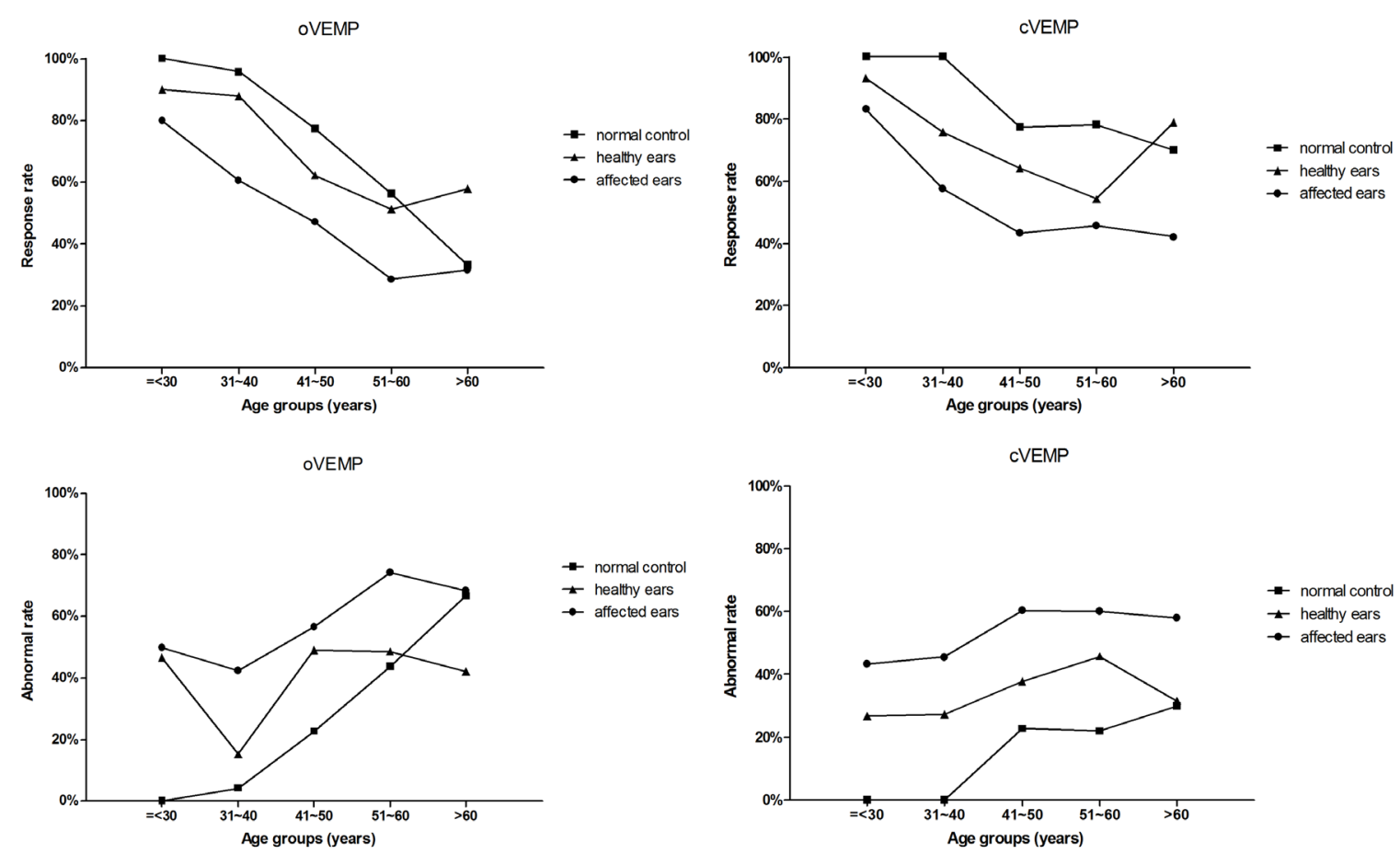

Figure 1. The effect of age on response and abnormal rates of VEMPs in affected, healthy and normal control ears. The response rates of cVEMP and oVEMP significantly declined with increasing age $\left(P<0.01, \chi^{2}\right.$ test $)$ in all affected, healthy and normal control ears. Abnormal rate of oVEMP in healthy ears rises significantly with age $\left(P<0.05, \chi^{2}\right.$ test).

Table 2. Response and abnormal rate of VEMPs between affected, healthy and normal control ears in every age group.

\begin{tabular}{|c|c|c|c|c|c|c|c|c|c|c|c|}
\hline \multirow{2}{*}{ Ears } & \multirow{2}{*}{ VEMPs } & \multicolumn{5}{|c|}{ Response rate (\%) } & \multicolumn{5}{|c|}{ Abnormal rate (\%) } \\
\hline & & Group 1 & Group 2 & Group 3 & Group 4 & Group 5 & Group 1 & Group 2 & Group 3 & Group 4 & Group 5 \\
\hline Affected ear & & $\begin{array}{c}83.3 \\
(n=30)\end{array}$ & $\begin{array}{c}57.6 \\
(n=33)\end{array}$ & $\begin{array}{c}43.4 \\
(n=53)\end{array}$ & $\begin{array}{c}45.7 \\
(n=35)\end{array}$ & $\begin{array}{c}42.1 \\
(n=19)\end{array}$ & 43.3 & 45.5 & 60.4 & 60.0 & 57.9 \\
\hline Healthy ear & cVEMP & $\begin{array}{c}93.3 \\
(\mathrm{n}=30)\end{array}$ & $\begin{array}{c}75.8 \\
(n=33)\end{array}$ & $\begin{array}{c}64.2 \\
(\mathrm{n}=53)\end{array}$ & $\begin{array}{c}54.3 \\
(\mathrm{n}=35)\end{array}$ & $\begin{array}{c}78.9 \\
(n=19)\end{array}$ & 26.7 & 27.3 & 37.7 & 45.7 & 31.6 \\
\hline Normal ear & & $\begin{array}{c}100.0 \\
(n=30)\end{array}$ & $\begin{array}{c}100.0 \\
(n=24)\end{array}$ & $\begin{array}{c}77.3 \\
(n=22)\end{array}$ & $\begin{array}{c}78.1 \\
(n=32)\end{array}$ & $\begin{array}{c}70.0 \\
(n=30)\end{array}$ & 0 & 0 & 22.7 & 21.9 & 30.0 \\
\hline$P$ & & 0.065 & $0.001^{\#}$ & $0.012^{*}$ & $0.021^{*}$ & $0.043^{*}$ & $0.000^{\#}$ & $0.001^{\#}$ & $0.005^{\#}$ & $0.007^{\#}$ & 0.115 \\
\hline Affected ear & & 80.0 & 60.6 & 47.2 & 28.6 & 31.6 & 50.0 & 42.4 & 56.6 & 74.3 & 68.4 \\
\hline Healthy ear & oVEMP & 90.0 & 87.9 & 62.3 & 51.4 & 57.9 & 46.7 & 15.2 & 49.1 & 48.6 & 42.1 \\
\hline Normal ear & & 100.0 & 95.8 & 77.3 & 56.3 & 33.3 & 0.0 & 4.2 & 22.7 & 43.8 & 66.7 \\
\hline$P$ & & $0.037^{*}$ & $0.002^{\#}$ & $0.043^{*}$ & $0.049^{*}$ & 0.161 & $0.000^{\#}$ & $0.001^{\#}$ & $0.027^{*}$ & $0.024^{*}$ & 0.161 \\
\hline
\end{tabular}

${ }^{*} P<0.05,{ }^{\#} P<0.01, \chi \chi^{2}$ test or Fisher's Exact Test.

with the age growing [14]. Similar results were observed in other studies, the prevalence of oVEMP in the over60 group was found significantly lower than that in the under-60 group. And the group over 60 years had significant longer latencies and smaller amplitudes compared with those under 60 years [18]. Piker et al. found that amplitudes were larger and thresholds were lower of both cVEMP and oVEMP in the younger people [19]. Decreased cVEMP and oVEMP amplitudes were also observed in older subjects (>50 years old) by Nguyen $e t$ al. [20]. The results above indicated that oVEMP and cVEMP changes with age increasing.

Considering the possible impact on VEMPs caused by age during clinical application, we divided SSHL patients into 5 age groups and investigated the features of otolithic dysfunction respectively. As shown in Table 2, in patients under 60 years, the response rates of cVEMP and oVEMP in affected ears were significantly lower and abnormal rates in affected ears were also significantly higher than those in the healthy and normal control 
ears $(P<0.05)$ (Table 2). But in people over 60 years old, response rates of cVEMP and oVEMP in normal ears were $70.0 \%$ and $33.3 \%$, decreased from $100 \%$ and $100 \%$ in normal people under 30 years old. And the response rates of cVEMP and oVEMP in SSHL affected ears in group 5 were $42.1 \%$ and 31.6\% respectively. Except the lower response rate of cVEMP in affected ears, the rest abnormalities of oVEMP and cVEMP were similar between affected, healthy and normal ears in group 5. Our results indicated that in SSHL patients under 60 years old the vestibular otolothic dysfunction could be detected definitely by using cVEMP and oVEMP tests. But VEMPs might not be able to reflect the real influence of otolithic function caused by SSHL truthfully in patients over 60 years old, especially oVEMP test. This might relate to the low response rate of VEMPs in older people. So we suggest that the reference value of ACS-VEMPs in assessing the otolithic function was limited in patients over 60 years old, maybe changing a more powerful stimulation method such as bone-conducted vibration (BCV) or electrical stimulation could be considered to solve this problem.

In patients under 30 years, the response rate of cVEMP in affected ears was $83.3 \%$, lower than the rate of $100 \%$ in normal ears, although there was no significant difference of cVEMP response rate in statistical analysis between affected and control ears. However, considering the thresholds elevated and latencies prolonged cVEMP waves, the cVEMP abnormal rate in affected ears was $43.3 \%$ in patients under 30 years, which was significantly higher than that in control ears. As to oVEMP, the absent rate was $20.0 \%$ and abnormal rate was $50.0 \%$ in affected ears in patients under 30 years. It illustrated that in most relatively young SSHL patients under 30 years old, the otolithic damage caused by SSHL might not be serious enough to be expressed in terms of absence of VEMP waves, the majority of the impairment could be shown in an obscure way which manifested as thresholds elevated or latencies prolonged VEMPs waves (Figure 2). This also suggested that the otolith organs might not be utterly destroyed easily in young SSHL patients.

As mentioned previously, varying degrees of abnormal VEMPs were observed in every SSHL age group under 60 years old. Moreover, in this study, the response rates of VEMPs significantly declined with increasing age, not only in normal control ears, but also in affected and contralateral healthy ears of SSHL patients (Figure 1).
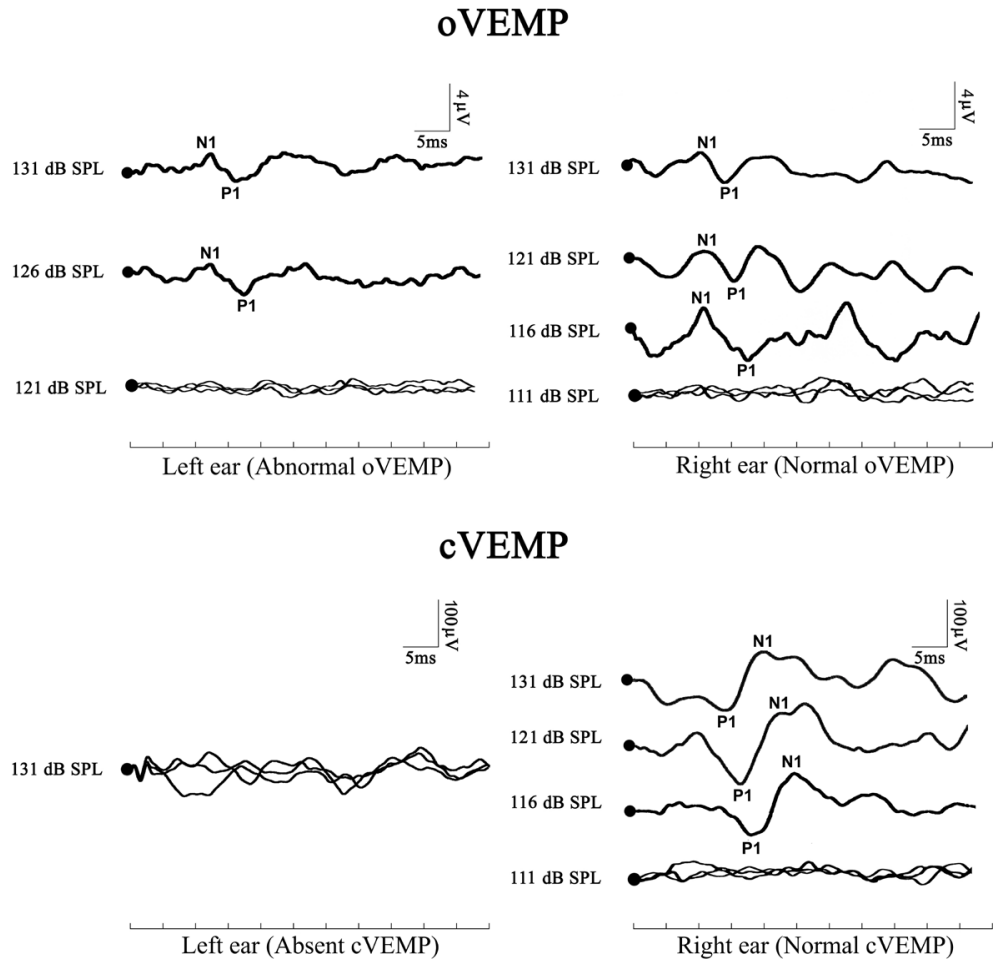

Figure 2. Typical case. A 17-year-old female patient, she has suffered from sudden hearing loss of the left ear with vertigo for 5 days. Her PTA (the average of thresholds on $500 \mathrm{~Hz}, 1$ $\mathrm{k} \mathrm{Hz}, 2 \mathrm{k} \mathrm{Hz}$ and $4 \mathrm{k} \mathrm{Hz}$ ) was $100 \mathrm{~dB}$ HL. The affected ear (left ear) showed abnormal oVEMP (elevated threshold and prolonged latencies) and absent cVEMP responses. Normal oVEMP and cVEMP waves were performed on the unaffected ear (right ear). 
It reminded us that, whether normal people or patients, we should consider the age effect during the results analysis in clinic application or scientific researches of VEMPs. And it was important to establish the normal data of VEMPs at all ages for reference. Although abnormal cVEMP and oVEMP showed an increasing trend in both affected and healthy ears, but there had no statistically significant differences between 5 age groups. The results above indicated that vestibular otolithic damage expressed by absent VEMPs was relatively less in younger patient than older patients with SSHL, but abnormal VEMPs behaved as thresholds elevated and latencies prolonged were more common in younger patients. Taking into account the abnormity of parameters, the abnormal rates of VEMPs showed no significant difference in affected ears of SSHL patients at different ages.

Many studies have focused on the features of vestibular involvement in SSHL, and tried to analyze the causes according to the pattern of the extent of vestibular lesions. But the causes of SSHL remain uncertain, as does the specific site of inner ear damage. Many treatments are used, including corticosteroids, antiviral drugs, and vasoactive and oxygen-based treatments. And a short course of oral high-dose corticosteroids are routinely recommend for primary treatment of SSHL [6]. There is much to learn about pathogenesis of SSHL, and more clinical trials are needed to establish evidence-based management.

\section{Conclusion}

In patients with SSHL under 60 years old, the vestibular otolothic dysfunction could be detected definitely by using ACS-cVEMP and ACS-oVEMP tests. But VEMPs might be unable to reflect the real influence of otolithic function caused by SSHL truthfully in patients over 60 years old, especially oVEMP test. Moreover, vestibular otolithic damage expressed by absent VEMPs was relatively less in younger patients, but abnormal VEMPs behaved as thresholds elevated and latencies prolonged were more common in younger patients. It is necessary to consider the age when the clinicians determine the otolithic condition of patients based on the VEMPs.

\section{Acknowledgements}

This study was supported by the National Natural Science Foundation of China, China (No. 3013300/C170703), Fundamental Research Funds for the Central Universities, China (No. 2012jdhz13), Shaanxi Major International Cooperative Project, China (No. 2013KW-28), and Key Science and Technology Program of Xi'an, China (No. SF1315 (1)).

\section{References}

[1] Stachler, R.J., Chandrasekhar, S.S., Archer, S.M., Rosenfeld, R.M., Schwartz, S.R., Barrs, D.M., Brown, S.R., Fife, T.D., Ford, P., Ganiats, T.G., Hollingsworth, D.B., Lewandowski, C.A., Montano, J.J., Saunders, J.E., Tucci, D.L., Valente, M., Warren, B.E., Yaremchuk, K.L. and Robertson, P.J. (2012) Clinical Practice Guideline: Sudden Hearing Loss. Otolaryngology_Head and Neck Surgery, 146, S1-S35. http://dx.doi.org/10.1177/0194599812436449

[2] Nosrati-Zarenoe, R., Arlinger, S. and Hultcrantz, E. (2007) Idiopathic Sudden Sensorineural Hearing Loss: Results Drawn from the Swedish National Database. Acta Oto-Laryngologica, 127, 1168-1175. http://dx.doi.org/10.1080/00016480701242477

[3] Wu, C.-S., Lin, H.-C. and Chao, P.-Z. (2006) Sudden Sensorineural Hearing Loss: Evidence from Taiwan. Audiology \& Neurotology, 11, 151-156. http://dx.doi.org/10.1159/000091198

[4] Tarnish, M., Katayama, N., Uchida, Y., Tominaga, M. and Nakashima, T. (2007) Thirty-Year Trends in Sudden Deafness from Four Nationwide Epidemiological Surveys in Japan. Acta Oto-Laryngologica, 127, 1259-1265. http://dx.doi.org/10.1080/00016480701242410

[5] Michel, O. (2011) The Revised Version of the German Guidelines "Sudden Idiopathic Sensorineural Hearing Loss". Laryngo-Rhino-Otologie, 90, 290-293. http://dx.doi.org/10.1055/s-0031-1273721

[6] Schreiber, B.E., Agrup, C., Haskard, D.O. and Luxon, L.M. (2010) Sudden Sensorineural Hearing Loss. The Lancet, 375, 1203-1211. http://dx.doi.org/10.1016/S0140-6736(09)62071-7

[7] Editorial Board of Chinese Journal of Otorhinolaryngology Head and Neck Surgery, The Otolaryngology Head and Neck Surgery Branch of Chinese Medical Association (2015) Guideline of Diagnosis and Treatment of Sudden Deafness. Chinese Journal of Otorhinolaryngology Head and Neck Surgery, 50, 443-447.

[8] Rauch, S.D. (2008) Idiopathic Sudden Sensorineural Hearing Loss. The New England Journal of Medicine, 359, 833840. http://dx.doi.org/10.1056/NEJMcp0802129

[9] Zhang, Q., Hu, J., Xu, X., Chen, Y., Zhang, Y., Wei, J., Zhang, Q., Xu, M. and Kaga, K. (2013) Objective Evaluation 
of Otolithic End Organs in Sudden Sensorineural Hearing Loss Patients. Chinese Journal of Otorhinolaryngology Head and Neck Surgery, 48, 389-393.

[10] Fujimoto, C., Egami, N., Kinoshita, M., Sugasawa, K., Yamasoba, T. and Iwasaki, S. (2015) Involvement of Vestibular Organs in Idiopathic Sudden Hearing Loss with Vertigo: An Analysis Using oVEMP and cVEMP Testing. Clinical Neurophysiology, 126, 1033-1038. http://dx.doi.org/10.1016/j.clinph.2014.07.028

[11] You, T.-Z., Wang, S.-J. and Young, Y.-H. (2014) Registering Grades of Sudden Deafness to Predict the Hearing Outcome via an Inner-Ear Test Battery. International Journal of Audiology, 53, 153-158. http://dx.doi.org/10.3109/14992027.2013.851798

[12] Papathanasiou, E.S., Murofushi, T., Akin, F.W. and Colebatch, J.G. (2014) International Guidelines for the Clinical Application of Cervical Vestibular Evoked Myogenic Potentials: An Expert Consensus Report. Clinical Neurophysiology, 125, 658-666. http://dx.doi.org/10.1016/j.clinph.2013.11.042

[13] Curthoys, I.S. (2010) A Critical Review of the Neurophysiological Evidence Underlying Clinical Vestibular Testing Using Sound, Vibration and Galvanic Stimuli. Clinical Neurophysiology, 121, 132-144. http://dx.doi.org/10.1016/j.clinph.2009.09.027

[14] Zhang, Q., Xu, X., Niu, X., Hu, J., Chen, Y. and Xu, M. (2014) Aging Effects on Air-Conducted Sound Elicited Ocular Vestibular-Evoked Myogenic Potential and Cervical Vestibular-Evoked Myogenic Potential. Chinese Journal of Otorhinolaryngology Head and Neck Surgery, 49, 897-901.

[15] Sheykholeslami, K., Murofushi, T. and Kaga, K. (2001) The Effect of Sternocleidomastoeid Electrode Location on Vestibular Evoked Myogenic Potential. Auris Nasus Larynx, 28, 41-43. http://dx.doi.org/10.1016/S0385-8146(00)00091-2

[16] Zhang, Q., Song, H., Hu, J., Chen, Y., Zhang, Y., Du, X., Zhang, Q., Wei, J., Xu, M. and Kaga, K. (2012) Characteristics of the Air-Conducted Ocular Evoked Myogenic Potential in the Young Normal Chinese Subjects. Chinese Journal of Otorhinolaryngology Head and Neck Surgery, 47, 15-18.

[17] Korres, S., Stamatiou, G.A., Gkoritsa, E., Riga, M. and Xenelis, J. (2011) Prognosis of Patients with Idiopathic Sudden Hearing Loss: Role of Vestibular Assessment. The Journal of Laryngology \& Otology, 125, 251-257. http://dx.doi.org/10.1017/S0022215110002082

[18] Chang, C.-M., Young, Y.-H. and Cheng, P.-W. (2012) Age-Related Changes in Ocular Vestibular-Evoked Myogenic Potentials via Galvanic Vestibular Stimulation and Bone-Conducted Vibration Modes. Acta Oto-Laryngologica, 132, 1295-1300. http://dx.doi.org/10.3109/00016489.2012.708437

[19] Piker, E.G., Jacobson, G.P., Burkard, R.F., McCaslin, D.L. and Hood, L.J. (2013) Effects of Age on the Tuning of the cVEMP and oVEMP. Ear and Hearing, 34, e65-e73. http://dx.doi.org/10.1097/aud.0b013e31828fc9f2

[20] Nguyen, K.D., Welgampola, M.S. and Carey, J.P. (2010) Test-Retest Reliability and Age-Related Characteristics of the Ocular and Cervical Vestibular Evoked Myogenic Potential Tests. Otology \& Neurotology, 31, 793-802. http://dx.doi.org/10.1097/MAO.0b013e3181e3d60e 